\title{
EFECTIVIDAD BIOLÓGICA DEL AZOXYSTROBIN PARA EL CONTROL DE Pyricularia oryzae Cav. Y Cercospora oryzae Miyake. EN ARROZ DE TEMPORAL EN VERACRUZ, MÉXICO 1
}

\author{
Enrique Becerra ${ }^{2}$, Oscar Tosquy ${ }^{2}$
}

\begin{abstract}
RESUMEN
Efectividad biológica del Azoxystrobin para el control de Pyricularia oryzae Cav. y Cercospora oryzae Miyake en el cultivo de arroz de temporal en Veracruz, México. El estado de Veracruz, México, siembra anualmente 22000 ha de arroz de temporal con un rendimiento medio de 3,5 t/ha, debido principalmente a problemas de sequía lo cual favorece la presencia de hongos como Pyricularia oryzae Cav. (quema del arroz) y Cercospora oryzae Miyake. (Mancha angosta). Con el objeto de conocer el comportamiento de nuevos fungicidas se evaluó la eficacia del Azoxystrobin en el control de estos hongos. El experimento se estableció en el municipio de Tres Valles, Veracruz, durante el temporal de 1999, con semilla de Milagro Filipino Depurado. El diseño estadístico utilizado fue bloques al azar con cuatro repeticiones. Los tratamientos evaluados fueron: Azoxystrobin a 0,2, 0,4 y 0,6 l/ha vs Tecto60 a $0,5 \mathrm{~kg} / \mathrm{ha}$ y un testigo sin aplicación. Estos fueron aplicados al presentarse los primeros síntomas de las enfermedades. Se evaluó la incidencia, número de lesiones en 20 plantas, índice de intensidad, el rendimiento de grano y fitotoxicidad. Se encontró que el Azoxystrobin obtuvo mejores resultados que el Tecto 60 y éste a su vez que el testigo no tratado. El mejor control para P. oryzae y C. oryzae y el mayor rendimiento de grano $(4432 \mathrm{~kg} / \mathrm{ha})$ se logró cuando se aplicó Azoxystrobin en dosis de 0,6 1/ha, aunque con 0,2 y 0,4 $1 /$ ha de este fungicida tuvo un buen control de estas enfermedades. Ninguno de los fungicidas causó toxicidad al arroz.
\end{abstract}

\begin{abstract}
Biologic Evaluation of the Azoxystrobin to control Pyricularia oryzae Cav. and Cercospora oryzae Miyake in crop of rice in Veracruz, Mexico. In the state of Veracruz, Mexico, around 22000 ha of rice are sown annually; the average of yield is about 3.5 t/ha and it is mainly due to drought problems, which favours the presence of the fungi Pyricularia oryzae Cav. (rice blast) and Cercospora oryzae Miyake (narrow spot). In order to know the behavior of new fungicides, the effectiveness of Azoxystrobin on the control of these fungi was evaluated. The experiment was established in the municipality of Tres Valles, Veracruz, at the beginning of the 1999 rainy season. Seed of the rice variety Milagro Filipino Depurado was used. The experimental design was a Randomized Complete Block design with four replications. The evaluated treatments were: Azoxystrobin at 0.2, 0.4 and $0.6 \mathrm{l} / \mathrm{ha}$ vs Tecto60 at $0.5 \mathrm{~kg} / \mathrm{ha}$ and a non-treated control.These treatments were applied when the first symptoms of rice blast and/or narrow spot were noticed. Factors being evaluate were: incidence, number of lesions in 20 plants, intensity index, phytotoxicity and grain yield. Azoxystrobin controlled both diseases better than Tecto 60; on the other hand, rice plants treated with Tecto 60 had significantly less lesions than the non-treated plants. The best control of P. oryzae and C. oryzae and the highest grain yield $(4432 \mathrm{~kg} / \mathrm{ha})$ were obtained when Azoxystrobin was applied at $0.6 \mathrm{l} / \mathrm{ha}$, although a good control of both diseases was also attained at 0.2 and $0.4 \mathrm{l} / \mathrm{ha}$. None of the fungicides caused toxicity to rice.
\end{abstract}

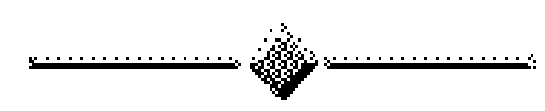

\section{INTRODUCCIÓN}

En México, el arroz es un cultivo de gran importancia, por ser uno de los granos alimenticios básicos, aporta poco más del $15 \%$ del promedio nacional de calorías, lo cual se debe a un consumo per cápita de siete kg. La producción de arroz palay obtenida a nivel nacional en 1998 fue de 458112 toneladas en una superficie de siembra de 109238 ha (INEGI, 1999), el estado de Veracruz es el primer productor con el 39\% del volumen total cosechado. En esta entidad, el arroz se produce bajo dos condiciones de cultivo: las siembras

\footnotetext{
1 Presentado en la XLVI Reunión Anual del PCCMCA en Puerto Rico, 2000.

2 Campo Experimental Cotaxtla-INIFAP. Apartado Postal 429, Veracruz, Ver. México. C.P. 91700
} 
de riego, que ocupan una superficie cercana a las 5000 ha con un rendimiento medio de entre 7,5 y 8 t/ha y las siembras de temporal que ocupan una superficie aproximada de 25000 ha con un rendimiento medio inferior a las $4 \mathrm{t} / \mathrm{ha}$, debido a problemas de sequía, altas infestaciones de malezas, plagas y la presencia de fitopatógenos como los hongos Pyricularia oryzae Cav., que provoca la quema o avanamiento del arroz y Cercospora oryzae Miyake, que causa la mancha angosta (Mendoza y Pinto, 1985). Estos dos hongos pueden presentarse en cualquier etapa del cultivo a nivel de follaje y panícula. Gálvez y Castaño (1974) mencionan que $P$. oryzae causa pérdidas en rendimiento de grano de entre 10 a $95 \%$, dependiendo de las variedades empleadas y de las condiciones ambientales que prevalezcan en la zona. Para el caso del estado de Veracruz, Rosas (1992) reporta un abatimiento de la producción hasta del 30\% en siembras de temporal.

El desarrollo de variedades resistentes es el método más preferido para el control de $P$. oryzae (Correa et al., 1997); sin embargo, las variedades pierden su resistencia en períodos de uno a tres años después de su liberación. Para el caso de $C$. oryzae, la resistencia varietal como método de control provoca el desarrollo de nuevas razas de este patógeno, por lo que el uso de prácticas agronómicas como la aplicación de fungicidas son consideradas como una buena opción de manejo.

Trabajos realizados para el control de $P$. oryzae en Veracruz demostraron que el fungicida Benlate (Benomil) en dosis de 250 y $125 \mathrm{~g} / \mathrm{ha}$ en cuatro aplicaciones, presentó una diferencia a favor con respecto al testigo sin aplicación, con 1476 y $1083 \mathrm{~kg} / \mathrm{ha}$ de arroz palay, respectivamente. En otro estudio el mejor tratamiento fue el Hinosán 50 a razón de uno y $0,75 \mathrm{~kg} / \mathrm{ha}$ con una sola aplicación y comparado con el testigo sin aplicación, hubo diferencias en rendimiento de arroz palay de $1803 \mathrm{~kg} / \mathrm{ha}$ para la primera dosis y $1225 \mathrm{~kg} / \mathrm{ha}$ con la segunda (Fraire, 1976).

En el estado de Veracruz para el control de la "quema" o avanamiento del arroz en las siembras de temporal se recomienda la aplicación de 1 1/ha de Hinosán cuando aparezcan los primeros síntomas y si el daño avanza se sugiere aplicar la mezcla de $800 \mathrm{ml}$ de Hinosán + 200g de Promil por hectárea (Ayón y Esqueda, 1992).

Con el objeto de conocer el comportamiento de un nuevo fungicida que pueda llegar a representar una alternativa eficiente para el control de hongos en el cultivo de arroz y además que no generen resistencia por el abuso de un sólo producto, se evaluó la eficacia del fungicida Bankit en el control de P. oryzae y $C$. oryzae. Es- te fungicida cuyo ingrediente activo es el azoxystrobin, perteneciente al novedoso grupo de las estrobirulinas con un espectro amplio de acción y de bajo impacto ambiental y a la salud humana, ha sido probado en Venezuela por Peraza y Yépez (1997) con excelente control de hongos a dosis de $75 \mathrm{~g}$ de i.a./ha.

\section{MATERIALES Y MÉTODOS}

Se estableció un experimento en la localidad de los Naranjos, Municipio de Tres Valles, Veracruz, en la modalidad de temporal. La siembra se llevó a cabo mecánicamente en surcos a $30 \mathrm{~cm}$, el 24 de mayo de 1999. Se utilizó semilla de la variedad Milagro Filipino Depurado a una densidad de $100 \mathrm{~kg} / \mathrm{ha}$.

Para el control de las malezas se aplicó en postemergencia temprana la mezcla de 10 litros de Estampir (381 $\mathrm{g} / \mathrm{l}$ de propanil $+40 \mathrm{~g} / \mathrm{l}$ de triclopyr) $+1,3$ litros de Gamit 480 CE (480 g/l de clomazone) en 400 litros de agua por hectárea. Posteriormente se realizaron deshierbes manuales para eliminar plantas de zacate de agua (Echinochloa colona), zacate cola de zorra (Leptochloa filiformis) y coquillo perenne (Cyperus rotundus).

La dosis de fertilización utilizada fue la 150-4646 , se aplicó todo el fósforo y potasio y 46 unidades de nitrógeno en forma de triple 17 el día 12 de julio y el resto del nitrógeno se aplicó en forma de urea el 21 de agosto. Por la presencia de algunas plagas como gusano soldado (Spodoptera frugiperda) y palomilla blanca (Rupella albinella) durante la etapa vegetativa y chinche café (Oebalus insularis) en floración se aplicó el insecticida Arrivo 200 en dosis de $250 \mathrm{ml} / \mathrm{ha}$.

Los tratamientos evaluados fueron cinco incluyendo un testigo comercial y uno sin aplicación cuyas dosis de producto comercial y de ingrediente activo se presentan en el Cuadro 1. El diseño experimental utilizado fue bloques al azar con cuatro repeticiones, las parcelas constaron de 12 surcos de $6 \mathrm{~m}$ de longitud, espaciados a $0,30 \mathrm{~m}$, teniendo como parcela útil los seis surcos centrales $\left(21,6 \mathrm{~m}^{2}\right)$

Cuadro 1. Tratamientos aplicados en el estudio de efectividad biológica del Azoxystobin en arroz. Primavera/verano $99 / 99$.

\begin{tabular}{lcc}
\hline \multicolumn{1}{c}{ Tratamiento } & Dosis kg o l/ha & g i.a./ha \\
\hline Azoxystobin (Bankit) & 0,200 & 50 \\
Azoxystobin (Bankit) & 0,400 & 100 \\
Azoxystobin (Bankit) & 0,600 & 150 \\
Tecto 60 & 0,500 & 300 \\
Testigo sin aplicar & --- & ---
\end{tabular}


Se realizó una sola aplicación de los tratamientos con una bomba de motor Robin R503 con boquilla de cono hueco, utilizando un volumen de agua equivalente a 300 1/ha y una cortina de plástico para evitar el arrastre del producto a otras parcelas. La aplicación se llevó a cabo el 9 de setiembre, a partir de que se presentaron los primeros síntomas de las enfermedades en follaje. El fungicida usado como testigo regional fue el Tecto 60 $(600 \mathrm{~g} / \mathrm{kg}$ de thiabendazole) en dosis de $500 \mathrm{~g} / \mathrm{ha}$.

El daño por $P$. oryzae y $C$. oryzae se evaluó sobre 20 macollas previamente marcadas antes de la aplicación de los tratamientos y a los 5,10 y 15 días después de la misma, con la finalidad de evaluar incidencia y mediante una escala de ocho grados estimar los daños de severidad de las enfermedades y así obtener el índice de intensidad (II) como lo mencionan French y Hebert (1982).

La escala de grados, para evaluar severidad (número de manchas) que se usó se describe a continuación :

$\begin{array}{ll}1=0 \% \text { de daño } & 2=1-15 \% \\ 3=16-30 \% & 4=31-45 \% \\ 5=46-60 \% & 6=61-75 \% \\ 7=76-90 \% & 8=91-100 \%\end{array}$

La fórmula para la obtención del índice de intensidad (I.I.):

1.1. $=\frac{\mathrm{n}(1)+\mathrm{n}(2)+\mathrm{n}(3)+\mathrm{n}(4)+\mathrm{n}(5)+\mathrm{n}(6)+\mathrm{n}(7)+\mathrm{n}(8)}{\sum n}$

donde $\mathrm{n}=$ número de observaciones de cada grado.

Otros de los parámetros evaluados fueron el rendimiento de grano expresado en kilogramos por hectárea al $14 \%$ de humedad y la fitotoxicidad (presencia de distorsión, amarillamiento o necrosis) mediante la siguien-

te escala en las mismas fechas de evaluación de enfermedades.

$1=0 \%$ de daño

$3=21-40 \%$ de daño

$5=61-100 \%$ de daño

Para homogeneizar las varianzas y dar una mejor interpretación a los resultados, los datos tomados fueron transformados tal como lo recomiendan Little y Jackson (1983), aunque por claridad se presentan los valores originales.

\section{RESULTADOS Y DISCUSION}

El muestreo preliminar para $P$. oryzae y $C$. oryzae hubo una mayor incidencia del primer hongo que del segundo y las enfermedades se presentaron más en unas parcelas que en otras, con un gradiente general de más a menos de la primera a la cuarta repetición, por lo que el diseño experimental utilizado fue adecuado. Cabe señalar que las condiciones ambientales no permitieron el buen desarrollo de estas enfermedades.

El análisis combinado de fechas de evaluación y tratamientos de las variables número de lesiones e índice de intensidad de P. oryzae y C. oryzae (Cuadro 2.) con el factor fechas de evaluación detectó diferencia altamente significativa para número de lesiones de $P$. ory$z a e$ y significativa para índice de intensidad de $P$. oryzae y número de lesiones de $C$. oryzae; para la fuente de variación tratamientos las diferencias fueron altamente significativas en todos los casos, lo que indica el diferente comportamiento que tuvieron los tratamientos en cada una de las variables de respuesta. La interacción fechas $x$ tratamientos resultó no significativa, lo cual se interpreta que en general los tratamientos se comportaron de manera similar en cada una de las fechas de eva-

Cuadro 2. Cuadrados medios y significancia del análisis combinado en las variables número de lesiones de $P$. oryzae y C. oryzae e índice de intensidad.

\begin{tabular}{lcccccccc}
\hline F.V. & G.L. & $\begin{array}{c}\text { Número lesiones } \\
\text { P. oryzae }\end{array}$ & $\begin{array}{c}\text { Indice intensidad } \\
\text { P. oryzae }\end{array}$ & $\begin{array}{c}\text { Número lesiones } \\
\text { C. oryzae }\end{array}$ & \multicolumn{2}{c}{$\begin{array}{c}\text { Indice intensidad } \\
\text { C. oryzae }\end{array}$} \\
\hline Repeticiones & 3 & 1.147 & $* *$ & $0,126 * *$ & 0,083 & $\mathrm{~ns}$ & 0,056 & $\mathrm{~ns}$ \\
Fechas de Evaluación & 2 & 0.434 & $* *$ & $0,052 *$ & 1,019 & $*$ & 0,058 & $\mathrm{~ns}$ \\
Error (a) & 6 & 0.027 & & 0,005 & 0,109 & & 0,063 & \\
Tratamientos & 4 & 1.024 & $* *$ & $0,076 * *$ & 0,512 & $* *$ & 0,190 & $* *$ \\
Fechas x tratamientos & 8 & 0.059 & $\mathrm{~ns}$ & $0,012 \mathrm{~ns}$ & 0,100 & $\mathrm{~ns}$ & 0,037 & $\mathrm{~ns}$ \\
Error (b) & 36 & 0.115 & & 0,015 & 0,051 & & 0,025 & \\
Total & 59 & & & & & & & \\
\hline
\end{tabular}

* = diferencias significativas en el análisis de varianza

** = diferencias altamente significativas en el análisis de varianza

ns $=$ no significancia en el análisis de varianza 
Cuadro 3. Prueba de Tukey al 5\% para el factor fechas de evaluación en el número de lesiones e índice de intensidad de P. oryzae y C. oryzae.

\begin{tabular}{cccrr}
\hline No. Fecha & $\begin{array}{c}\text { Días después de } \\
\text { la aplicación }\end{array}$ & $\begin{array}{c}\text { Lesiones de } \\
\text { P. oryzae }\end{array}$ & $\begin{array}{c}\text { Lesiones de } \\
\text { C. oryzae }\end{array}$ & $\begin{array}{c}\text { Indice de intensidad } \\
\text { P. oryzae }\end{array}$ \\
\hline & 5 & $* 3.947 \mathrm{a}$ & $* 2.323 \mathrm{a}$ & $* 1.835 \mathrm{~b}$ \\
2 & 10 & $2.675 \mathrm{~b}$ & $1.260 \mathrm{~b}$ & $1.820 \mathrm{~b}$ \\
3 & 15 & $3.536 \mathrm{a}$ & $1.000 \mathrm{~b}$ & $1.915 \mathrm{a}$ \\
\hline
\end{tabular}

* = valores máximos alcanzados.

Tratamientos con letras similares son estadísticamente iguales (Tukey al 5\%)

luación, por lo que existe independencia entre ambos factores.

La prueba de Tukey al 5\% para el factor fechas de evaluación corroboró las diferencias detectadas en los análisis de varianza, dándole validez estadística a las medias obtenidas (Cuadro 3). Tanto para P. oryzae como para $C$. oryzae la primera fecha de evaluación (5 días después de aplicados los tratamientos) presentó el mayor número de lesiones y se obtuvo el menor número de lesiones en la segunda fecha de evaluación para $P$. oryzae y en la tercera fecha de evaluación para $C$. ory$z a e$, siendo ésta última estadísticamente igual a la segunda.

En cuanto al índice de intensidad de $P$. oryzae los menores valores se obtuvieron en la segunda y primera fecha de evaluación.

En el factor tratamientos, Tukey al 5\% puso en superioridad estadística al testigo sin aplicación con el mayor número de lesiones promedio por planta con 5,45 y 2,35 para $P$. oryzae y $C$. oryzae respectivamente (Cuadro 4) de igual forma se comportó el índice de intensidad (con 1,99 y 1,76). Para el caso de la primera y tercera variable, el resto de los tratamientos fueron estadísticamente iguales, aunque se observa que el mejor control se obtuvo cuando se aplicó el Azoxystrobin con la dosis más alta, seguido del Azoxystrobin a 0,2 y 0,4 1/ha. En la segunda y cuarta variable los menores valo- res los presentó también el Azoxystrobin a 0,6 1/ha, aunque fue estadísticamente igual a las otras dosis de este mismo producto.

Referente a la variable rendimiento de grano, la Figura 1 muestra los resultados obtenidos por tratamiento, donde la mayor cifra la obtuvo el Azoxystrobin a dosis de $0,6 \mathrm{l} / \mathrm{ha}$ con un rendimiento cercano a los 4500 $\mathrm{kg} / \mathrm{ha}$, superando en 1021 y $1678 \mathrm{~kg}$ al Tecto 60 y testigo sin aplicación, respectivamente. El análisis de varianza arrojó diferencia altamente significativa para tratamientos, lo que indica que éstos tuvieron diferente respuesta en cuanto a rendimiento de grano. $\mathrm{Al}$ realizar los contrastes pertinentes (Cuadro 5) se detectó signifi-

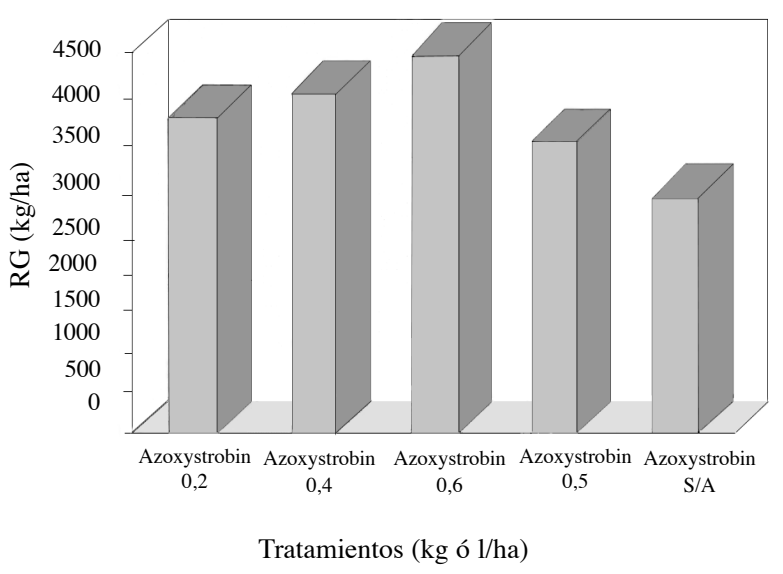

Figura 1. Rendimiento de grano obtenido en cada tratamiento.

Cuadro 4. Prueba de Tukey al $5 \%$ para el factor tratamientos en el número de lesiones e índice de intensidad de P. oryzae y C. oryzae.

\begin{tabular}{lcccc}
\hline $\begin{array}{l}\text { No. Tratamiento } \\
\text { (kg o I /ha) }\end{array}$ & $\begin{array}{c}\text { Lesiones de } \\
\text { P. oryzae }\end{array}$ & $\begin{array}{c}\text { Lesiones de } \\
\text { C. oryzae }\end{array}$ & $\begin{array}{c}\text { Indice intensidad } \\
\boldsymbol{P . ~ o r y z a e}\end{array}$ & $\begin{array}{c}\text { Indice intensidad } \\
\boldsymbol{P} \text { oryzae }\end{array}$ \\
\hline 1.- Azoxystrobin 0,2 & $2,86 \mathrm{~b}$ & $1,00 \mathrm{c}$ & $1,80 \mathrm{~b}$ & $1,49 \mathrm{bc}$ \\
2.- Azoxystrobin 0,4 & $2,87 \mathrm{~b}$ & $1,32 \mathrm{bc}$ & $1,82 \mathrm{~b}$ & $1,54 \mathrm{bc}$ \\
3.- Azoxystrobin 0,6 & $2,40 \mathrm{~b}$ & $1,00 \mathrm{c}$ & $1,80 \mathrm{~b}$ & $1,44 \mathrm{c}$ \\
4.- Tecto 0,5 & $3,32 \mathrm{~b}$ & $1,95 \mathrm{ab}$ & $1,85 \mathrm{ab}$ & $1,64 \mathrm{ab}$ \\
5.- Testigo S/A & $* 5,45 \mathrm{a}$ & $* 2,35 \mathrm{a}$ & $* 1,99 \mathrm{a}$ & $* 1,76 \mathrm{a}$ \\
\hline
\end{tabular}

* = valores máximos alcanzados

Tratamientos con letras similares son estadísticamente iguales (Tukey al 5\%). 
Cuadro 5. Cuadrados medios y significancia para contrastes ortogonales en la variable rendimiento de grano en cada uno de los tratamientos evaluados.

\begin{tabular}{lcrl}
\hline Fuente de variación & $\begin{array}{c}\text { Grados de } \\
\text { libertad }\end{array}$ & Cuadrado medio \\
\hline Bloques & 3 & 411781,34 & $\mathrm{~ns}$ \\
Tratamientos & 4 & 1582524,00 & $* *$ \\
C1: T5 vs T1,T2,T3, T4 & 1 & 4058554,50 & $* *$ \\
C2: T4 vs T1,T2,T3 & 1 & 1175315,00 & $*$ \\
C3: T3vs T1,T2 & 1 & 937730,69 & $*$ \\
C4: T2 vs T1 & 1 & 158484,50 & $\mathrm{~ns}$ \\
Error & 12 & 157009,33 & \\
Total & 19 & & \\
\hline
\end{tabular}

$*$ diferencias significativas en el análisis de varianza

$* *=$ diferencias altamente significativas en el análisis de varianza

ns $=$ no significancia en el análisis de varianza

cancia para los tres primeros, donde para el contraste 1 se asume que hubo respuesta a la aplicación de fungicidas, al controlar estas enfermedades con los agroquímicos se obtuvo un $40,9 \%$ más de rendimiento de grano (3880, 4 kg/ha) sobre el testigo sin aplicación. En el segundo contraste, la aplicación de Azoxystrobin presenta una diferencia a favor de $626 \mathrm{~kg} / \mathrm{ha}$ que resultó ser estadísticamente superior al Tecto 60 y para el caso del contraste tres la dosis más alta de Azoxystrobin con un rendimiento de $4432 \mathrm{~kg} /$ ha superó a la dosis intermedia y baja de este mismo fungicida. Finalmente en el cuarto contraste no se detectó significancia, lo que señala que el Azoxystrobin aplicado a 0,2 y 0,4 1/ha es igualmente eficiente desde el punto de vista estadístico en el control de enfermedades.

Al realizar los muestreos en los tratamientos no se encontraron efectos de fitotoxicidad en el cultivo de arroz con ninguno de los productos aplicados en las fechas evaluadas.

\section{CONCLUSIONES}

Con base a los resultados obtenidos y a la discusión que de ellos se hace, se derivan las siguientes conclusiones:

Todos los tratamientos a base de fungicidas superaron al testigo sin aplicación, el cual presentó el mayor número de lesiones e índice de intensidad de $P$. oryzae y C. oryzae y el menor rendimiento de grano (2754 $\mathrm{kg} / \mathrm{ha})$.

El Azoxystrobin controló mejor las dos enfermedades y superó en un 18,3\% al Tecto 60 , el cual presentó un rendimiento de grano de $3411 \mathrm{~kg} / \mathrm{ha}$.
El mejor control para $P$. oryzae y $C$. oryzae y el mayor rendimiento (4432 kg/ha) se logró cuando se utilizó Azoxystrobin en la dosis alta, aunque con las dosis de 0,2 y $0,41 /$ ha de este fungicida se tiene un excelente control de estas enfermedades.

Ninguno de los fungicidas o sus dosis ocasionaron toxicidad al cultivo de arroz.

\section{LITERATURA CITADA}

AYÓN, R.E.A.; ESQUEDA, V. E. 1992. Manual de producción de arroz en el estado de Veracruz. Folleto para productores No. 2. México, CECOT. CIRGOC. INIFAP. SARH. $17 \mathrm{p}$.

CORREA, V.F.; TULANDE, E.; RAISER, M.; ARICAPA, G.; PRADO, G. 1997. Caracterización de la reacción a Pyricularia grisea en variedades de arroz de América Latina. Selección de fuentes de resistencia. In X Conferencia Internacional de Arroz para América Latina y el Caribe. Araure, Venezuela. p. 92.

FRAIRE, M.R. 1976. Control químico del añublo del arroz (Pyricularia oryzae Cav.) en Veracruz. Informe interno. INIA - CIASE. Campo Cotaxtla. p.5-15.

FRENCH, E.R.; HEBERT, T.T. 1982. Métodos de Investigación Fitopatológica. San José, Costa Rica. IICA. p. 289.

GÁlvEZ, E.G.E.; CASTAÑO, J. 1974. Aplicación de productos quimioterapéuticos al suelo para el control de Pyricularia oryzae en arroz. Fitopatología 9:18-23.

INEGI (Instituto Nacional de Estadística, Geografía e Informática). 1999. El sector alimentario en México, Aguascalientes, México, INEGI. 312 p.

LITTLE, M.T.; JACKSON, F. 1983. Métodos estadísticos para la investigación en la agricultura. $4^{\text {ta }}$ reimpresión. México. D.F., Ed. Trillas. p. 136-137.

MENDOZA, Z.C.; PINTO, B. 1985. Principios de fitopatología y enfermedades causadas por hongos. In: Cincuentenario de la especialidad de Parasitología Agrícola. 1935-1985. Chapingo, México.

PERAZA, W.; YÉPEZ, G. 1997. Evaluación de la eficacia del fungicida Azoxystrobin en el control de piricularia ( $P y$ ricularia oryzae) en el cultivo de arroz (Oryza sativa). In: Memoria de la X Conferencia Internacional de Arroz para América Latina y el Caribe. Araure, Venezuela. p. 90.

ROSAS, G.X. 1992. Respuesta del arroz (Oryza sativa L.) de temporal a la fertilización mineral. Tesis de Ingeniero Agrónomo en Sistemas de Producción Agrícola. Instituto Teconológico Agropecuario (ITA), No. 18. V. Ursulo Galván, Ver. 90 p. 